\title{
FABRICATION AND CHARACTERIZATION OF 3D PRINTED, 3D MICROELECTRODE ARRAYS WITH SPIN COATED INSULATION AND FUNCTIONAL ELECTROSPUN 3D SCAFFOLDS FOR "DISEASE IN A DISH" AND “ORGAN ON A CHIP" MODELS

\author{
Nilab Azim ${ }^{1,2}$, Tariq Ausaf , $^{3}$, Avra Kundu ${ }^{1}$, Lei Zhai ${ }^{1,2}$ and Swaminathan Rajaraman, 3, 4, 5*
} ${ }^{1}$ NanoScience Technology Center (NSTC), ${ }^{2}$ Department of Chemistry, ${ }^{3}$ Department of Electrical \& Computer Engineering, ${ }^{4}$ Department of Material Science \& Engineering, University of Central Florida, Orlando, FL 32826, USA \\ ${ }^{5}$ Bridging the Innovation Development Gap (BRIDG), Neo City, FL 34744, USA
}

\begin{abstract}
We demonstrate a new fabrication technology for 3D Microelectrode Arrays (MEAs) to stimulate and record electrophysiological activity from cellular networks in-vitro. Electrospun Polyethylene Terephthalate (PET) 3D scaffolds are coupled to the fabricated MEAs which make them fully functional for "disease in a dish" and "organ on a chip" models to promote cell/tissue growth and regeneration. The microfabrication technology involves $3 \mathrm{D}$ towers realized by $3 \mathrm{D}$ printing and a metallization layer, defined by stencil mask evaporation techniques. Multiple insulation strategies are reported: a drop-casted/spincoated 3D layer of Polystyrene (PS) and an evaporated layer of $\mathrm{SiO}_{2}$, both of which are laser micromachined to realize the $3 \mathrm{D}$ microelectrodes.
\end{abstract}

\section{INTRODUCTION}

Microelectrode array (MEA) technology is a highly popular and widely used platform for recording and stimulating electrical activity in electrogenic cells such as neurons, cardiomyocytes, pancreatic beta cells etc. for both in vitro and in vivo applications [1]. In MEA electrophysiology, the quality of the extracellular recording of electrical signals strongly depends on the interfacial contact between the electrogenic cells and the microelectrodes. Additionally, spike activity from a neuron can only be detected up to a distance of $<100 \mu \mathrm{m}$ from the planar surface [1]. Thus, the distance between the isopotential source and electrode is typically in the range of $50-100 \mu \mathrm{m}$, making it essential for the microelectrodes to have high spatial resolution, which is achieved utilizing electrodes that are typically $\sim 30 \mu \mathrm{m}$ in diameter with small inter-electrode distances $(200 \mu \mathrm{m})$. Microfabrication technologies are apt for realizing such designs and thus 3D MEAs have primarily been realized with silicon microassembly or complex glass-based approaches [2]. This however restricts monolithic MEA fabrication technologies to be predominantly two-dimensional (2D) since lithographic techniques on non-planar surfaces is particularly challenging [3], [4]. Absence of 3D functionality in MEAs without complex processes makes them inadequate to capture signals that occur at a certain height when cultures mature to obtain a 3D form. Additionally, the realization of a suitable insulation layer for such 3D electrodes has always remained a challenge due to diverse topographies for conformal deposition of biocompatible materials with a low thermal budget.

Tissue-specialized extracellular matrix (ECM) plays a key role in tissue homeostasis, cell attachment, growth, proliferation, differentiation, morphology, polarization, directional motility, migration and cell spreading [5]. As the tissue environment is essentially three-dimensional (3D), there is an increasing need to extend cell culture matrices, support scaffolds and microelectrodes to $3 \mathrm{D}$ as well. While some cells are able to synthesize all required ECM components, others require an external source, particularly when grown in serum-free culture. Therefore the synthetic ECM must mimic the extracellular environment of the host as best as possible [6]. This would reduce the large mismatch between chemical, biomechanical and textural properties of cells and synthetic interfacing devices such as MEAs. As a result, a more stable and functionally predictable interfacial interaction between the cell and its environment, especially ECM, has a profound effect on cell phenotype and fate. Therefore, the chemical, topographical and elastomechanical properties of non-biological substrate surfaces that are supposed to be in intimate contact with cells or tissue needs careful engineering.

Additive manufacturing has recently shown the potential to transform microengineering with its ability to realize true $3 \mathrm{D}$ structures monolithically. Such additive manufacturing involves a layer by layer construction of the designed 3D geometry using a 3D printer. Among the various 3D printing technologies, stereolithography (SLA) based 3D printing provides a balance between the print resolution, print time, build volume and cost. SLA $3 \mathrm{D}$ printers are inexpensive, benchtop systems typically found in makerspaces, which are providing a growing alternative to clean rooms for realization of nanobiosensors, biomedical micro-electromechanical systems (BioMEMS) and micro-total analysis systems (MicroTAS) [7]. We have recently introduced the concept of 'Makerspace Microfabrication' [7] which was used for the realization of biological microdevices such as 2D Microelectrode arrays (MEAs), microneedles (MNs) and Microfluidic channels (MFCs). Specifically, the process described in our prior work was enabled by 3D printing, Ink Casting, Lamination and Micromachining and involved a close synergy between the additive and subtractive micromachining processes. The process described was hierarchical in nature and each subsequent process built upon the functionality provided by the preceding procedure. Due to the highly flexible and adaptive nature of 'Makerspace Microfabrication' new processes offering advanced functionalities can be added to realize other biological microdevices such as 3D MEAs. The realization of an appropriate insulation layer and a synthetic ECM material atop 3D topographies is challenging but can be addressed by adding suitable toolboxes such as benchtop dropcasting/spin-coating, and electrospinning to 'Makerspace Microfabrication'.

In this paper, we report the first application of $3 D$ printing toward the microfabrication of $3 D M E A s$ that are vital to the understanding of cellular models of excitable cells in a 3D microenvironment in-vitro mimicking in-vivo like conditions. The metallization of the $3 \mathrm{D}$ printed parts has been performed by standard physical vapor deposition techniques through a micromilled stencil mask. Two insulating strategies have been demonstrated for the metallized 3D electrodes. The first employs a drop-casted, spin-on 3D layer of Polystyrene (PS) while the second involves evaporated $\mathrm{SiO}_{2}$, both of which have subsequently been laser micromachined to realize the recording sites on the $3 \mathrm{D}$ printed tower. Non-woven, polyethylene terephthalate nanofiber scaffolds (PET NFS) were 
prepared by electrospinning technology on the laser micromachined $3 \mathrm{D}$ electrodes to mimic the fibrous proteins in native extracellular matrix towards constructing a synthetic 3D scaffold. Full spectrum impedance analysis of the fabricated electrodes confirms microelectrode behavior and Fourier Transform Infrared Spectroscopy (FTIR) analysis confirms the presence of both a dropcasted/spin-on layer and electrospun layer to be PS and PET respectively. SEM imaging has also been performed to demonstrate the geometry of the 3D printed electrode, the conformal deposition of the PS insulating layer and the PET NFS.

\section{MATERIALS AND METHODS}

The 3D MEAs were designed in Solidworks (2016 x64 bit edition, Dassault Systems Inc., Waltham, MA, USA). The MEA chip has a size of $49 \mathrm{~mm} \times 49 \mathrm{~mm} \times 1 \mathrm{~mm}$ to ensure connectivity with the Multi Channel Systems (Reutlingen, Aspenhaustrasse, Germany) recording amplifiers. Two patches, each containing ten recording sites in the form of $3 \mathrm{D}$ towers were designed. The microtowers had a base diameter of $250 \mu \mathrm{m}$ and a height of $400 \mu \mathrm{m}$. The designed CAD file was directly printed in a 3D SLA printer Form Labs Form 2 (Somerville, MA, USA) with a laser wavelength of $405 \mathrm{~nm}$ using a photopolymer clear resin (FLGPCL04, Formlabs, Somerville, MA, USA). The metallization of the 3D MEAs and definition of the traces $(200 \mu \mathrm{m}$ wide) are performed by $\mathrm{Ti} / \mathrm{Au}$ deposition through a micromilled stainless steel stencil mask. For the fabrication of the stainless steel mask a 90-degree T-8 Mill Tool $(150 \mu \mathrm{m}-250 \mu \mathrm{m}$ diameter; T-Tech, Peachtree Corners, GA, USA) was spun at 55,000 rpm in a T-Tech J5 Quick Circuit Prototyping Systems to cut into a stainless steel sheet $(80 \mu \mathrm{m}$ thick; Trinity Brand Industries, Countryside, IL, USA). In order to define the metallization layer, titanium ( $\mathrm{Ti}, 4 \mathrm{~N} 5$ purity pellets) and gold ( $\mathrm{Au}$, $5 \mathrm{~N}$ purity pellets) [Kurt J. Lesker, Jefferson Hills, Pennsylvania, USA] were deposited by electron-beam (E-beam) evaporation [Thermionics Laboratory Inc., Hayward, California, USA]. The Ti and $\mathrm{Au}$ layers were deposited in a vacuum of $3.1 \times 10^{-6}$ Torr to a thickness of $10 \mathrm{~nm}$ at a deposition rate of $1.0 \mathrm{~nm} / \mathrm{s}$ and $100 \mathrm{~nm}$ at $1.0 \mathrm{~nm} / \mathrm{s}$, respectively. A biocompatible laminate layer (Medco®RTS3851-17 adhesives $\sim 50 \mu$ m thick plus poly ethylene terephthalate (PET) $\sim 20 \mu \mathrm{m}$ thick; Medco Coated Products, Cleveland, $\mathrm{OH}, \mathrm{USA}$ ) is subsequently bonded to the 3D printed chip to insulate the traces thereby enabling realization of 3D tower MEAs with electrodes having a size of the entire $3 \mathrm{D}$ printed structure. The biocompatible laminate is additionally micromilled prior to its alignment and attachment to have openings corresponding to the size of the two patches of 3D tower arrays, each containing ten recording sites. A culture well having an inner diameter of $30 \mathrm{~mm}$ is $3 \mathrm{D}$ printed and bonded using a biocompatible adhesive (Epo-tek ${ }^{\circledR}$ $353 \mathrm{ND}$ ) to realize the final device. Figure 1 (a) depicts an exploded view of the components of the 3D MEAs along with close-up schematic of the ten recording sites in a single patch.

To realize smaller electrodes, an insulation layer is defined atop of the 3D microelectode towers [Figure 2(b)]. In the first strategy, the insulation layer was achieved by a drop-casting/spin-coating technique using polystyrene (PS). The insulation layer via dropcasting/spin-coating was obtained using a polymer solution prepared from $10 \%(\mathrm{w} / \mathrm{v})$ PS ( $280 \mathrm{kDa}$, Sigma Aldrich, St. Louis, Missouri, USA) dissolved in tetrahydrofuran (THF) (Thermo Fisher Scientific, Waltham, Massachusetts, USA). Approximately $2 \mathrm{~mL}$ of $10 \%$ PS in THF solution was drop-casted to fully cover the MEA device and allowed to sit at room temperature for the solvent to evaporate to leave behind a thin film layer. The spin-coated insulation layer was defined in the same fashion: $2 \mathrm{~mL}$ of $10 \%$ PS was drop-casted and immediately spun before the solvent could evaporate. The solution was spun at $500 \mathrm{rpm}$ for 1 minute, followed by drying at room temperature for approximately 10 minutes. In the second strategy, a manually rotated e-beam evaporation of $\mathrm{SiO}_{2}$ pellets [4N5 purity from Kurt J. Lesker, Jefferson Hills, Pennsylvania, USA] was performed. The deposition rate was 10 $\mathrm{nm} / \mathrm{s}$ with a thickness of $400 \mathrm{~nm}$. These two strategies were used to define the uniform 3D insulation layer which can subsequently be selectively defined by laser micromachining [Figure 2 (c); electrodes: $30 \mu \mathrm{mx} 30 \mu \mathrm{m}$; a $532 \mathrm{~nm}$ laser beam at an energy level of $1.2 \mathrm{~mJ}$ ] utilizing QuickLaze 50ST2 (Eolite Lasers, Portland, OR, USA).
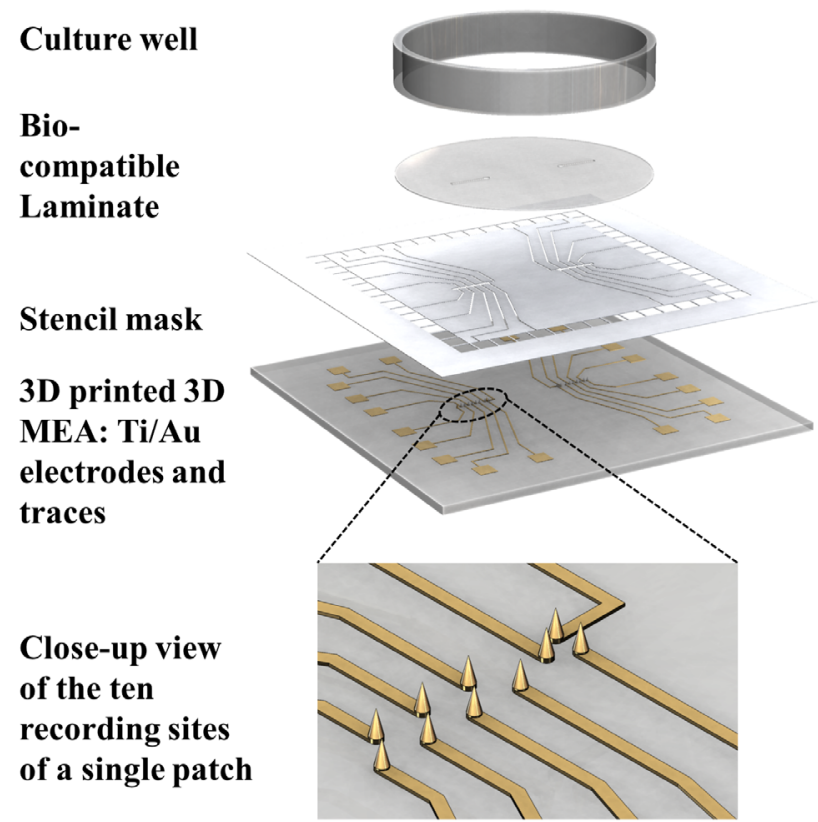

Figure 1: Exploded view of the 3D MEA: $3 D$ microtowers, metallization, laminate insulation and containment culture well with a close-up of the ten recording sites per patch.

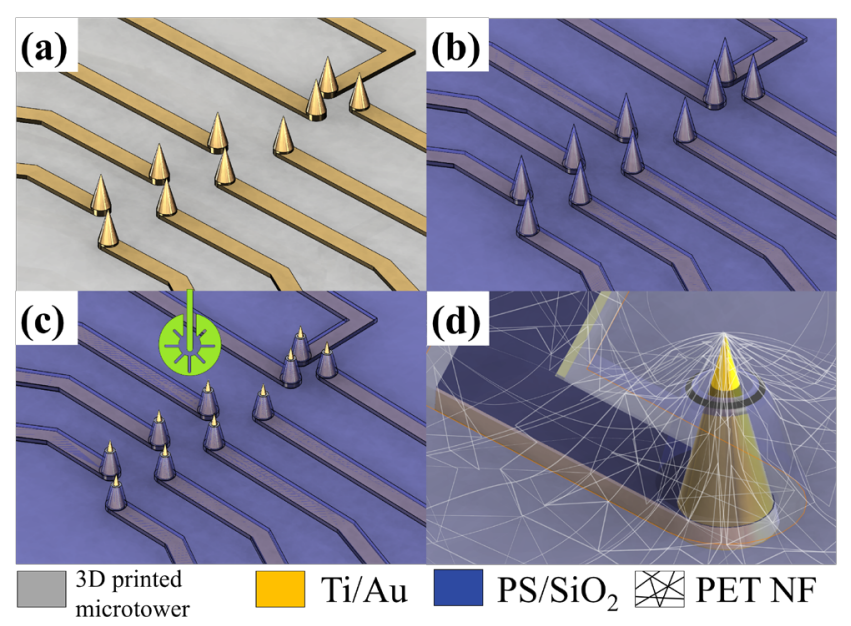

Figure 2: $3 D$ insulation and scaffolding process flow - (a) Metallized 3D towers; (b) PS/SiO $\mathrm{S}_{2}$ insulation atop the metallized $3 \mathrm{D}$ towers; (c) Insulation ablation process (Laser symbol in green); (d) PET nanofiber scaffold (NFS) deposited on the insulated, laser ablated, 3D MEA.

The polyethylene terephthalate (PET) nanoscaffold [Figure 2 (d)] can optionally be defined atop either insulation layer using an 
electrospinning process. This technique utilizes an electric field to produce a polymer jet from a solution [8], [9]. The polymer jet is formed when the surface tension of the solution is surpassed by the applied electrostatic charge. This jet dries during flight towards the grounded collector and is elongated by electrostatic repulsion with nearby segments of the same jet. By the time the jet reaches the collector, the solvent has evaporated and the jet has solidified into a nanofiber. Critical parameters such as the polymer of interest's molecular weight, concentration of polymer, solution properties, electrical potential between the needle and the collector, flow rate of the solution, the needle gauge and distance between the needle and the collector need to be optimized in order to achieve nanofibers with specific properties. In our work, 3D scaffolds were fabricated from PET nanofibers. The polymer solution was composed of $20 \%$ (w/w) PET (Sigma Aldrich, St. Louis, Missouri, USA) dissolved in a mixture of trifluoroacetic acid (TFA) (Thermo Fisher Scientific, Waltham, Massachusetts, USA) and dichloromethane (DCM) (Thermo Fisher Scientific, Waltham, Massachusetts, USA) having a TFA: DCM volume ratio of 70:30. Electrospinning of PET was performed using a working distance of $12 \mathrm{~cm}$, an applied voltage of $10 \mathrm{kV}$ and a flow rate of $99 \mu \mathrm{L} / \mathrm{hr}$. Figures 3 (a) and (b) show schematics of the drop-casting/spin-coating and electrospinning processes respectively.

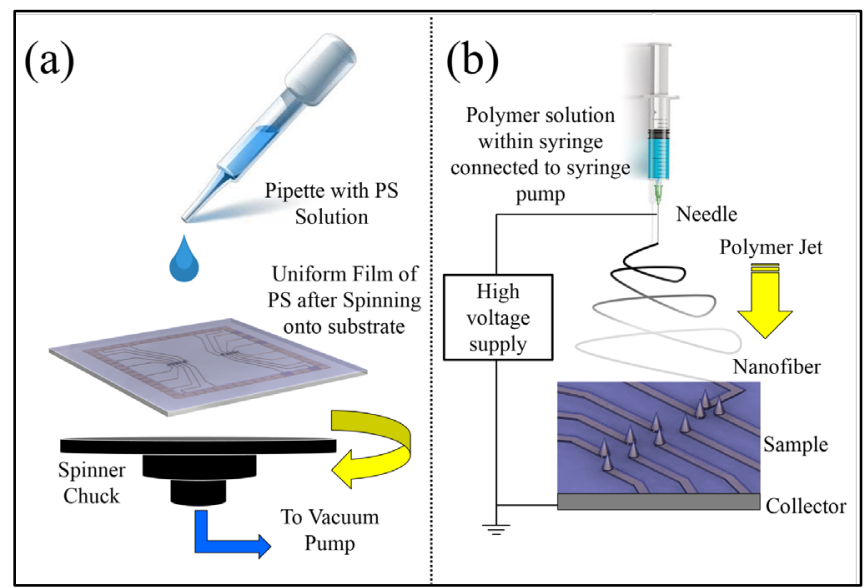

Figure 3: (a) Schematic of the drop-casting and (b) electrospinning techniques, both on $3 D$ microtower arrays.

\section{RESULTS AND DISCUSSIONS}

Figure 4 shows the optical micrographs of routed, metallized microtowers with the packaged 3D MEAs depicted in the inset. At this stage the entire 3D printed tower is metallized and the size of the microelectrode corresponds to that of the 3D printed tower (base diameter: $250 \mu \mathrm{m}$ and height: $400 \mu \mathrm{m})$. The biocompatible transparent lamination layer protects the traces from any culture solution contained in the culture well and as a result from shorting.

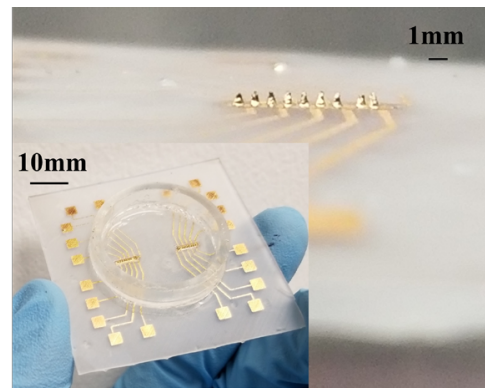

Figure 4: 3D tower MEA: final, packaged device shown in the inset.
Figure 5 shows the SEM image of the PS insulated, metallized $3 \mathrm{D}$ microtower. It is observed that when the PS solution is dropcasted on the 3D printed structure, the PS forms a blanket layer atop the 3D towers as shown in Figure 5 (a) upon drying. A closer look at this image additionally reveals micropores at the base of the towers, formed due to the drop-casted PS solution being thicker around the base of the towers and during the drying of the PS solution, bubbles of the solvent (THF), escape from the PS layer leading to the observed porosity. This issue however is completely resolved when the samples are spin coated after drop casting. The PS layer is uniform and conformal with the $3 \mathrm{D}$ printed tower geometry [Figure $5(\mathrm{~b})$ ].

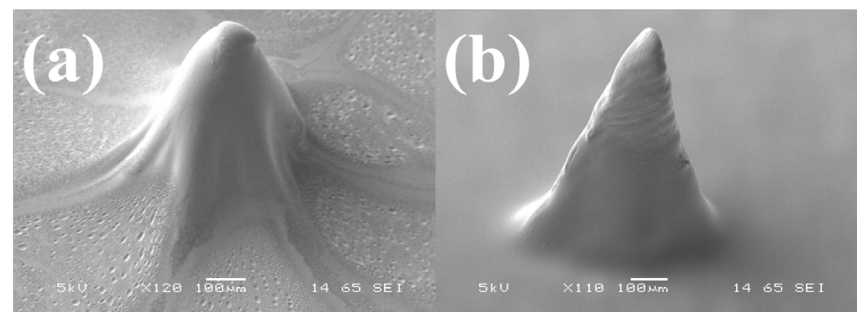

Figure 5: SEM image of 3D tower MEA coated with PS: (a) after drop-casting and (b) drop-casted and spin-coated.

Figure 6 (a) depicts a close-up SEM image of the 3D tower MEA after drop-casting and spin-coating of PS insulation. The recording site in the 3D MEA was defined by laser micromachining and the laser ablated electrode is shown in Figure 6 (b).

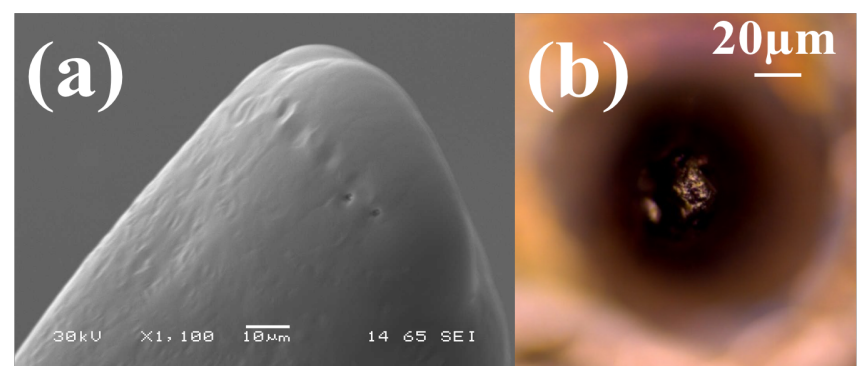

Figure 6: (a) SEM image of 3D tower tip after drop-casting and spin-coating of PS layer and (b) PS ablated $3 D$ tower showing a recording site corresponding to a $30 \mu \mathrm{m} \times 30 \mu \mathrm{m}$ size.

Figure 7 (a) shows the SEM image of 3D PET nanoscaffold deposited on $\mathrm{SiO}_{2}$ insulation. It is clearly observed that the nanofibers interlink between the 3D electrodes providing effective scaffolding. Figure 7 (b) shows the close-up of the PET NFS with a fiber width of $\sim 200-500 \mathrm{~nm}$.

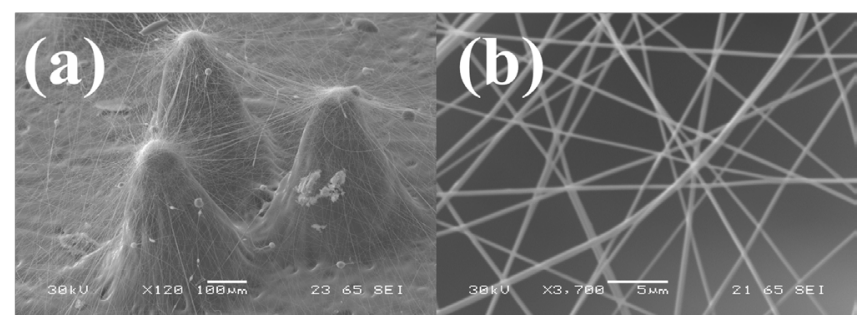

Figure 7: (a) SEM image of 3D PET NFS on $\mathrm{SiO}_{2}$ insulation and (b) Close-up view of the NFS showing fiber width of $\sim 200-500 \mathrm{~nm}$.

Figure 8 shows the FTIR analysis of the electrospun and dropcasted/spin-coated 3D layers with signature peaks of PET and PS respectively. A Spectrum 100 FTIR spectrometer (Perkin Elmer, 
Waltham, Massachusetts, USA) was used to obtain the data. Some noteworthy peaks from the PS FTIR are: the $695 \mathrm{~cm}^{-1}$ peak from a ring bend indicating that the molecule is monosubstituted and the $748 \mathrm{~cm}^{-1}$ peak from C-H wag signifying a C-H bond to a benzene ring, both of which are characteristic peaks of polystyrene. One noteworthy peak for PET was the $726 \mathrm{~cm}^{-1}$ peak, which derives from the benzene ring's interaction with polar ester groups. The $\mathrm{C}-\mathrm{O}$ peaks at $1265 \mathrm{~cm}^{-1}, 1247 \mathrm{~cm}^{-1}$ peak and $1101 \mathrm{~cm}^{-1}$ and the very prominent carbonyl peak at $1715 \mathrm{~cm}^{-1}$, confirm the presence of the ester groups from PET. Finally, the peak at $2961 \mathrm{~cm}^{-1}$ helps confirm the presence of a methylene group to suggest that the molecule is PET.

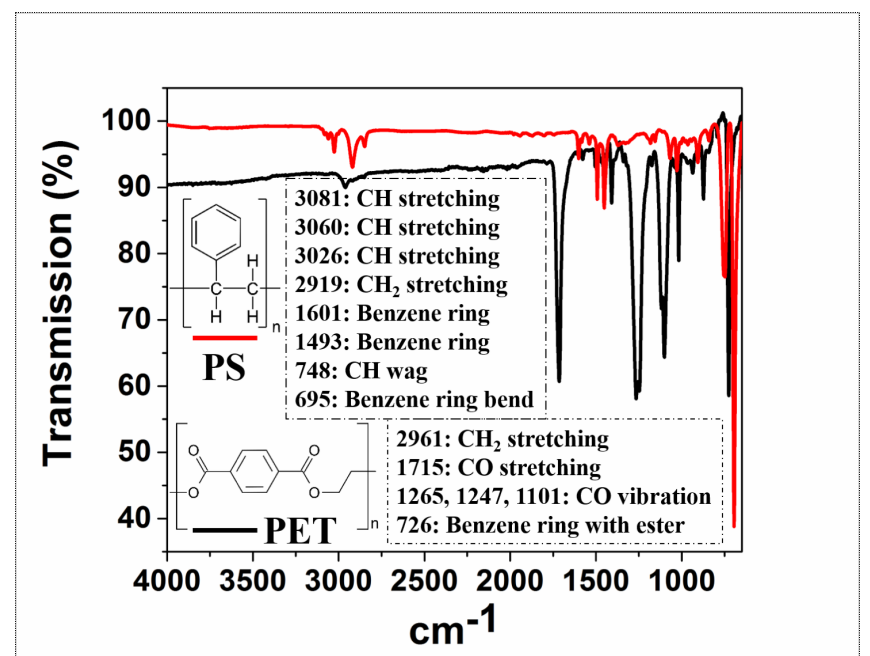

Figure 8: FTIR analysis of the spin coated and electrospun 3D MEA sample showing the distinct signature peaks for various components of both PET and PS respectively.

Impedance measurements of the MEAs were performed with the final device using Bode 100 (Omicron Labs, Houston, TX, USA) with Dulbecco's Phosphate Buffer Solution (Thermo Fisher Scientific, Waltham, MA, USA) as the electrolyte. The impedance scans were carried out from $10 \mathrm{~Hz}$ to $1 \mathrm{MHz}$ with a platinum wire (eDAQ, Denistone East, Australia) as the counter electrode. Table 1 depicts the impedance and phase characteristics of the metallized 3D towers, $\mathrm{SiO}_{2}$ ablated 3D MEA with scaffolding and PS ablated insulation showing comparable properties to others reported values in literature [10], [11].

Table 1: Summary of real part and phase of impedance of the various $3 D$ MEAs developed at electrophysiologically relevant frequencies.

\begin{tabular}{|c|c|c|c|c|c|c|}
\hline \multirow{2}{*}{ MEA type } & \multicolumn{3}{|c|}{ Impedance (Ohms) } & \multicolumn{3}{c|}{ Phase (degrees) } \\
\cline { 2 - 7 } & $100 \mathrm{~Hz}$ & $1 \mathrm{kHz}$ & $10 \mathrm{kHz}$ & $100 \mathrm{~Hz}$ & $1 \mathrm{kHz}$ & $10 \mathrm{kHz}$ \\
\hline $3 \mathrm{D}$ microtower & $6.7 \mathrm{k}$ & $5.3 \mathrm{k}$ & $4.1 \mathrm{k}$ & -50 & -15 & -10 \\
\hline $\begin{array}{c}\mathrm{SiO}_{2} \text { laser } \\
\text { micromachining }\end{array}$ & $80.2 \mathrm{k}$ & $52.1 \mathrm{k}$ & $12.2 \mathrm{k}$ & -15 & -12 & -10 \\
\hline $\begin{array}{c}\text { PS laser } \\
\text { micromachining }\end{array}$ & $47.6 \mathrm{k}$ & $36.8 \mathrm{k}$ & $10.3 \mathrm{k}$ & -20 & -15 & -10 \\
\hline
\end{tabular}

\section{CONCLUSIONS}

Our fabrication process is capable of rapidly realizing 3D MEAs which are highly versatile and fully functional for in-vitro applications. With a union between makerspace microfabrication coupled with standard microfabrication technologies as needed, we have demonstrated the realization of 3D tower MEAs of base dimeter $=250 \mu \mathrm{m}$ and height $=400 \mu \mathrm{m}$. The electrodes sizes can be reduced after either evaporating a $\mathrm{SiO}_{2}$ layer or drop-casting/spincoating of PS solution, both of which can subsequently be laser micromachined. Additional development of biocompatible 3D scaffolds atop the 3D MEAs provides for a device ready for "disease in a dish" and "organ on a chip" applications of cell/tissue growth, proliferation and long-term cultures in-vitro.

\section{REFERENCES}

[1] R. Kim, S. Joo, H. Jung, N. Hong, and Y. Nam, "Recent trends in microelectrode array technology for in vitro neural interface platform," Biomed. Eng. Lett., vol. 4, no. 2, pp. 129-141, Jun. 2014.

[2] P. Ruther et al., "The NeuroProbes Project-Multifunctional Probe Arrays for Neural Recording and Stimulation," Proc. 13th Annu. Conf. IFESS, Sept.21-25, Freiburg, Ger., vol. 53, pp. 238-240, 2008.

[3] S. Rajaraman, S.-O. Choi, M. A. McClain, J. D. Ross, M. C. LaPlaca, and M. G. Allen, "Metal-TransferMicromolded Three-Dimensional Microelectrode Arrays for in-vitro Brain-Slice Recordings," $J$. Microelectromechanical Syst., vol. 20, no. 2, pp. 396-409, Apr. 2011.

[4] S. Rajaraman et al., "Microfabrication technologies for a coupled three-dimensional microelectrode, microfluidic array," J. Micromechanics Microengineering, vol. 17, no. 1, pp. 163-171, Jan. 2007.

[5] A. Blau, "Cell adhesion promotion strategies for signal transduction enhancement in microelectrode array in vitro electrophysiology: An introductory overview and critical discussion," Curr. Opin. Colloid Interface Sci., vol. 18, no. 5, pp. 481-492, Oct. 2013.

[6] P. Zorlutuna et al., "Microfabricated Biomaterials for Engineering 3D Tissues," Adv. Mater., vol. 24, no. 14, pp. 1782-1804, Apr. 2012.

[7] A. Kundu, T. Ausaf, and S. Rajaraman, "3D Printing, Ink Casting and Micromachined Lamination (3D PICL $\mu \mathrm{M}$ ): A Makerspace Approach to the Fabrication of Biological Microdevices," Micromachines, vol. 9, no. 2, p. 85, Feb. 2018.

[8] J. Lannutti, D. Reneker, T. Ma, D. Tomasko, and D. Farson, "Electrospinning for tissue engineering scaffolds," Mater. Sci. Eng. C, vol. 27, no. 3, pp. 504-509, Apr. 2007.

[9] N. Bhardwaj and S. C. Kundu, "Electrospinning: A fascinating fiber fabrication technique," Biotechnol. Adv., vol. 28, no. 3, pp. 325-347, May 2010.

[10] C. Nick, S. Yadav, R. Joshi, J. J. Schneider, and C. Thielemann, "A three-dimensional microelectrode array composed of vertically aligned ultra-dense carbon nanotube networks," Appl. Phys. Lett., vol. 107, no. 1, p. 13101, Jul. 2015.

[11] G. S. Guvanasen et al., "A Stretchable Microneedle Electrode Array for Stimulating and Measuring Intramuscular Electromyographic Activity," IEEE Trans. Neural Syst. Rehabil. Eng., vol. 25, no. 9, pp. 1440-1452, Sep. 2017.

\section{CONTACT}

*Swaminathan Rajaraman; Ph:+1-407-823-4339; Email: Swaminathan.Rajaraman@,ucf.edu 